\title{
Study of Chaos in the Buck Converter
}

\author{
Enric Fossas and Gerard Olivar
}

\begin{abstract}
A de-de buck converter controlled by naturallysampled, constant-frequency pulsewidth modulation in continuous conduction mode gives rise to a great variety of behavior, depending on the values of the parameters of the circuit. We analyze the one-periodic and two-periodic orbits which cross the voltage ramp once per cycle, and we study their stability by computing the characteristic multipliers associated with each one. Subharmonics, bifurcations, and the presence of a strange attractor are also studied. A plot of the number of crossings in the ramp is drawn. This becomes a helpful tool for investigating the evolution of the trajectories when they are close to the attractor. When analytic computations are impossible, we resort to numerical algorithms to simulate the orbits.
\end{abstract}

\section{INTRODUCTION}

$\mathbf{T}$ HERE are two main reasons for studying unstable and chaotic circuits. Taking the traditional view that instability is unacceptable, it is important to know if this sort of behavior can be present in an experimental situation in order to avoid it. From a more optimistic view, if a mode of operation is well understood and clarified, it can be of engineering use. The understanding of chaotic behavior opens up new possibilities of operating regimes that can help to optimize design.

The main antecedent in the study of chaos in power electronic circuits can be found in the work reported almost simultaneously by Deane and Hamill, on the one hand [1], [2], and Wood on the other [3]. The three papers illustrate how chaos can occur in dc-to-dc switching converters of the buck type operating in the continuous conduction mode and being controlled by a pulsewidth modulator (PWM). Dc-to-dc switching converters of the buck type are widely used in a large area of applications - ranging from switching converters for telecommunications to airborne radar power supplies-due to their high efficiency and simplicity of control using PWM techniques.

This paper reexamines (Section II) the buck regulator studied by Deane and Hamill in [1], [2] and analyzes the oneperiodic and two-periodic orbits which cross the modulator voltage ramp once per cycle (Section III). Subsequently, the orbits stability is studied by computing their respective characteristic multipliers and a strange attractor is detected (Section IV). The dynamics of the chaotic zone are then analyzed using the number of crossings in the modulator ramp (Section V). As in [1], the numerical simulations are performed with the following parameter values (see Fig. 1): $L=20 \mathrm{mH}$, $C=47 \mu F, R=22 \Omega, a=8.4, V_{\text {ref }}=11.3 \mathrm{~V}, V_{L}=3.8 \mathrm{~V}$, $V_{U}=8.2 \mathrm{~V}$, and $T=400 \mu \mathrm{s}$.

Manuscript received March 31, 1994; revised October 21, 1994 and June 16, 1995. This paper was recommended by Associate Editor M. P. Kennedy. The authors are with the Department of Applied Mathematics and Telematics, Polytechnical University of Catalonia, Barcelona 08034, Spain.

Publisher Item Identifier S 1057-7122(96)00732-5.

\section{MODELING}

Unlike the classical examples that have been proved to show chaotic dynamics, such as Lorenz's equations or Chua's circuit, different behavior can be expected in the buck converter due to its inherent discontinuities. Fig. 1 shows the block diagram of a buck regulator that uses a PWM voltage loop. We assume throughout that the components in the circuit are ideal $^{1}$. The comparator $A_{2}$ has infinite gain, the switches $S$ and $S^{\prime}$ have zero on, and infinite off resistance, and can switch instantly. During the interval when switch $S$ is on, $S^{\prime}$ is off and the input provides energy to the load as well as to the inductor. During the interval when switch $S$ is off, the inductor current, which flows through switch $S^{\prime}$, transfers some of its stored energy to the load. One of the methods for controlling the output voltage employs switching at a constant frequency (hence, a constant switching time period $T=t_{o n}+t_{\text {off }}$ ), and adjusting the on-duration of the switch to control the average output voltage. In this method, called pulsewidth modulation (PWM) switching, the switch duty ratio $d$, which is defined as the ratio of the on-duration to the switching time period, is varied. In order to avoid the discontinuous conduction mode, and, at the same time, to allow the existence of light load levels, we assume that the switches allow bi-directional current flow [4], and are turned on and off in a complementary fashion.

Considering that the linear amplifier $A_{1}$ has gain $a$, we can write

$$
v_{c o}(t)=a\left(v(t)-V_{\text {ref }}\right) .
$$

Then, both $v_{c o}$ and $v_{\text {ramp }}$, the voltage of the ramp, are applied to the comparator, and every time the output difference changes its sign, the position of the switches $S$ and $S^{\prime}$ is commuted in such a way that $S$ is open (and $S^{\prime}$ is closed) when the control voltage exceeds the ramp voltage; otherwise $S$ is closed (and $S^{\prime}$ is open). Due to the fact that the discontinuous conduction mode does not take place, the converter can be represented by a piecewise-linear vector field, described by two systems of differential equations as follows:

System 1: $v_{c o}(t)>v_{\text {ramp }}(t)$

$$
\frac{d}{d t}\left(\begin{array}{c}
v(t) \\
i(t)
\end{array}\right)=\left(\begin{array}{cc}
-1 /(R C) & 1 / C \\
-1 / L & 0
\end{array}\right)\left(\begin{array}{c}
v(t) \\
i(t)
\end{array}\right)
$$

System 2: $v_{c o}(t)<v_{\text {ramp }}(t)$

$$
\frac{d}{d t}\left(\begin{array}{c}
v(t) \\
i(t)
\end{array}\right)=\left(\begin{array}{cc}
-1 /(R C) & 1 / C \\
-1 / L & 0
\end{array}\right)\left(\begin{array}{c}
v(t) \\
i(t)
\end{array}\right)+\left(\begin{array}{c}
0 \\
V_{i n} / L
\end{array}\right)
$$

${ }^{1}$ In this paper, no parasitic effects have been considered. If they are to be taken into account, the methodology described in [10]-[12] should be followed 


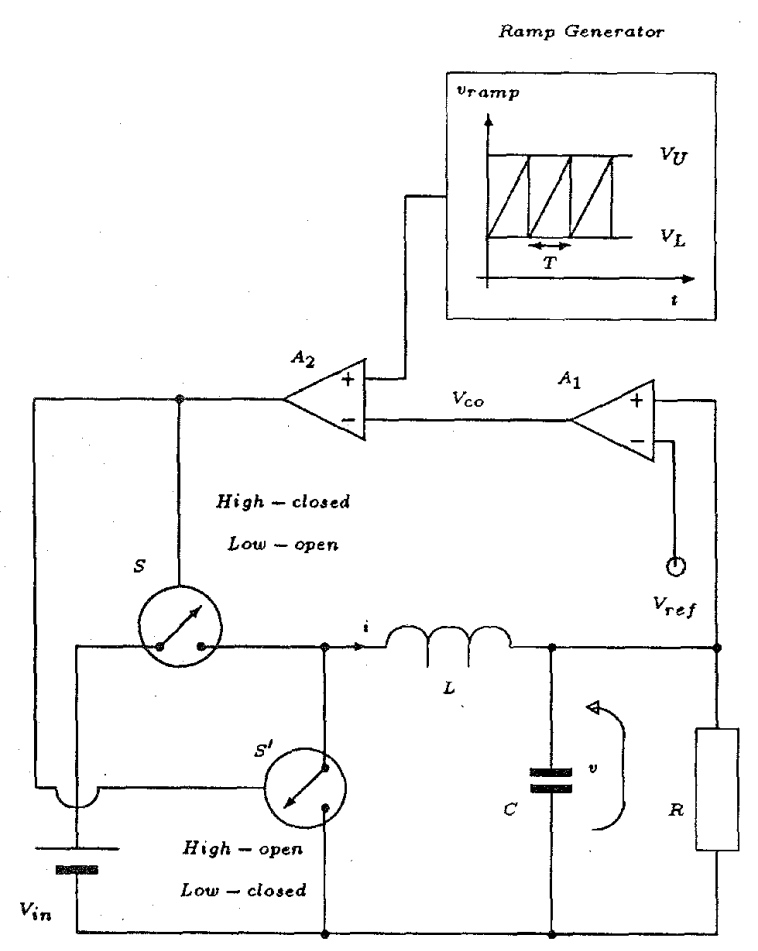

Fig. 1. Block diagram of the buck converter.

where $v$ is the voltage through the capacitor and $i$ is the intensity of the current in the inductor. Using the notation $x=(v, i)^{\mathcal{T}}$, (we denote $y^{T}$ the transpose of $y$ ), (2) and (3) can be combined in only one linear expression

$\frac{d x}{d t}=f(x, t) \equiv\left(\begin{array}{cc}-1 /(R C) & 1 / C \\ -1 / L & 0\end{array}\right) x(t)+\left(\begin{array}{c}0 \\ V_{i n} / L\end{array}\right) \mathbf{I}_{\mathcal{S}}(t)$

being

$$
\mathbb{1}_{\mathcal{S}}(t)= \begin{cases}0 & \text { if } t \notin \mathcal{S} \\ 1 & \text { if } t \in \mathcal{S}\end{cases}
$$

with

$$
\mathcal{S}=\left\{t \geq 0: v_{c o}<v_{\text {ramp }}(t)\right\}
$$

The voltage of the ramp is given by

$$
v_{\text {ramp }}(t)=V_{L}+\left(V_{U}-V_{L}\right) t / T
$$

where $V_{L}$ and $V_{U}$ are respectively the lower and upper voltages of the ramp and $T$ its period. Observe that the time, $t$, explicitly appears in the expression of $f$, concretely in the term $\mathbf{I}_{\mathcal{S}}(t)$, that somehow represents the control ramp. Due to the explicit appearance of $t$, the system is nonautonomous. If we fix a set of initial conditions $v_{0}=v\left(t_{0}\right)$ and $i_{0}=i\left(t_{0}\right)$, as the system of differential equations is linear, we will be able to compute exactly the solution of each one.

Let us write

$$
k=\frac{1}{2 R \bar{C}}, \quad \omega=+\sqrt{\frac{1}{L C}-k^{2}}
$$

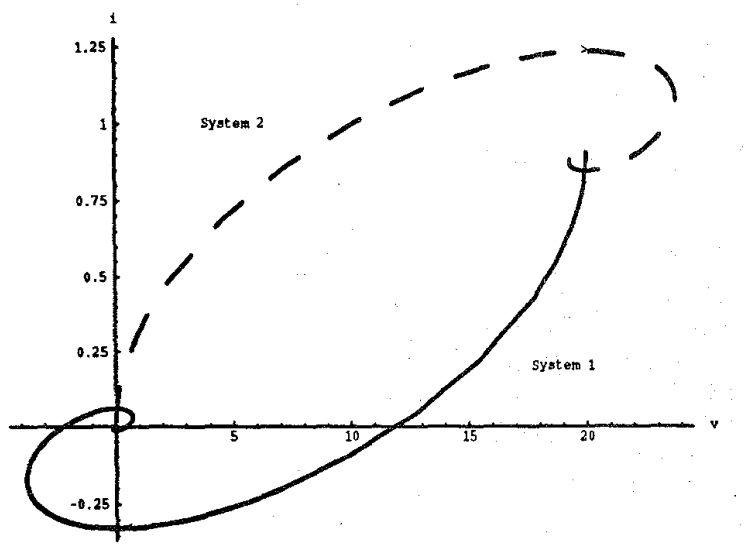

Fig. 2. Orbit of systems 1 and 2 around its equilibrium point in the state space $\left(V_{i n}=20\right)$. The solid orbit corresponds to system 1 and the dashed one to system 2 .

and supposing that

$$
\frac{1}{L C}-k^{2}>0
$$

we can define the real matrix

$$
A=\left(\begin{array}{cc}
-k / \omega & 1 /(C \omega) \\
-1 /(L \omega) & k / \omega
\end{array}\right)
$$

and we have the following solutions for the systems:

System 1: $v_{c o}(t)>v_{\text {ramp }}(t)$

$\left(\begin{array}{c}v(t) \\ i(t)\end{array}\right)=e^{-k\left(t-t_{0}\right)}\left[I \cos \omega\left(t-t_{0}\right)+A \sin \omega\left(t-t_{0}\right)\right]\left(\begin{array}{c}v_{0} \\ i_{0}\end{array}\right)$

System 2: $v_{c o}(t)<v_{\text {ramp }}(t)$

$$
\begin{aligned}
& \left(\begin{array}{c}
v(t) \\
i(t)
\end{array}\right)=\left(\begin{array}{c}
V_{i n} \\
V_{i n} / R
\end{array}\right)+ \\
& e^{-k\left(t-t_{0}\right)}\left[I \cos \omega\left(t-t_{0}\right)+A \sin \omega\left(t-t_{0}\right)\right]\left(\begin{array}{c}
v_{0}-V_{i n} \\
i_{0}-V_{i n} / R
\end{array}\right)
\end{aligned}
$$

where $I$ is the identity matrix. These expressions of the solutions are equivalent to those obtained in [1] and [2]. It follows that, between two commutation consecutive times, we know exactly the state variables of the system. Essentially, they are a combination of exponential and sinusoidal functions.

We next study the stability of each system.

For system $1\left(v_{c o}(t)>v_{\text {ramp }}(t)\right)$, we have an equilibrium point $\bar{x}=(\bar{v}, \bar{i})^{\mathcal{T}}=\overrightarrow{0}$, and if we find the eigenvalues of $D f(\bar{x})$, we obtain

$$
\lambda_{1}=-k+j \omega, \quad \lambda_{2}=-k-j \omega .
$$

As $-k<0, \bar{x}$ is an asymptotically stable equilibrium point and the orbits around it will be like the solid curve in Fig. 2. If we make similar computations for system 2, the equilibrium point we have now is $\left(V_{i n}, V_{i n} / R\right)$, with the same eigenvalues. So, the orbits around it will be very similar to the one computed for system 1 , as it is plotted with a broken line in Fig. 2 . 


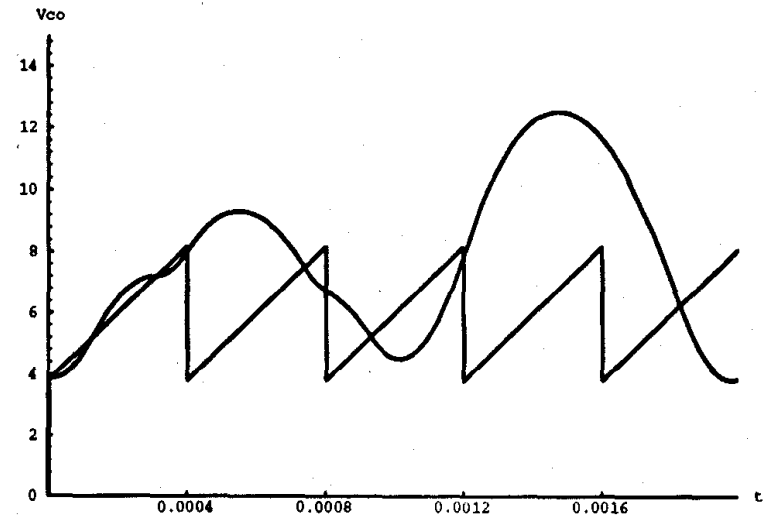

Fig. 3. Typical trajectory of the control voltage.

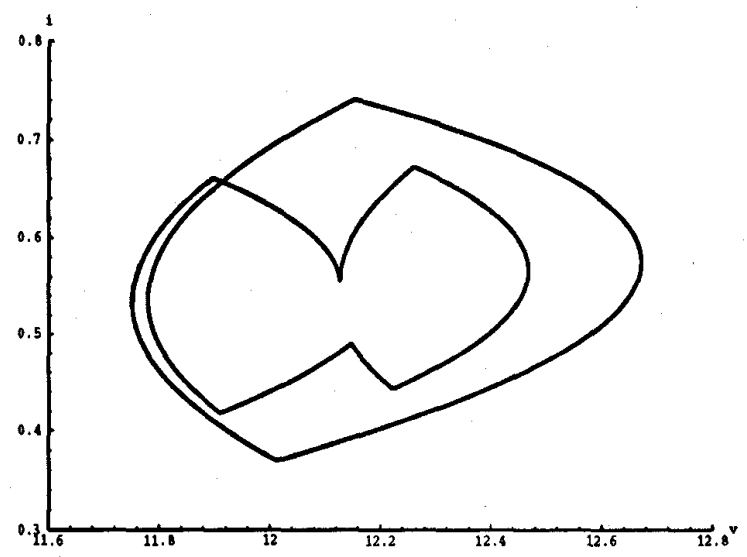

Fig. 4. Typical trajectory in the phase space.

Thus, the evolution of each system, considered separately, is trivial, and corresponds to damped oscillations around the equilibrium point. But when both systems are taken together as a global one, as in the buck converter, the behavior is radically different. When a trajectory is near the equilibrium point of system 1 , one has $v_{c o}<v_{\text {ramp }}$, the circuit switches its topology and the orbit is attracted to the equilibrium point of system 2, because this is the system that is working. When the trajectory, attracted to the equilibrium point of system 2 , moves near it, system 1 begins to work, attracting the trajectory to the equilibrium point of system 1 . This wandering between the two equilibrium points of the separate systems produces a highly nontrivial evolution, without any equilibrium point in the global system. In the range of values of the parameters suggested in [1] and [2], a typical trajectory of the control voltage is shown in Fig. 3. In the phase space $(v, i)$, the same trajectory is drawn in Fig. 4.

Notice that, as shown in Fig. 3, multiple pulsing can occur, where the switches change the topology of the circuit many times per ramp cycle. This is very undesirable in practice as it greatly increases the switching losses. One way to avoid it is to use a latch. With a latched PWM, multiple pulsing is eliminated but subharmonics and chaos are still possible [5].

We have represented the solutions of the global system both in the phase plane $(v, i)$ (see Fig. 4 ), and in the control

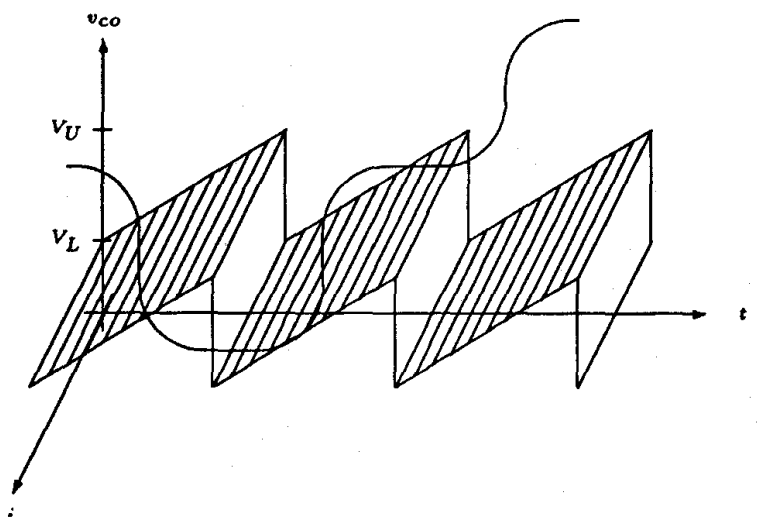

Fig. 5. A curve in the tridimensional space $\left(t, i, v_{c o}\right)$.

plane $\left(t, v_{c o}(t)\right)$ together with the ramp (see Fig. 3). As this is a nonautonomous system, if we project the trajectory in the phase space the time information is lost (obviously, the projection in the control plane does not include the $i$-variable and then information is lost, too). If we want to see how the voltage and current are changing with time, we need a tridimensional representation $(t, v(t), i(t))$. When increasing $t \geq 0$, the curve described in the space gives the solution of the system starting at $v_{0}=v(0)$ and $i_{0}=i(0)$ as initial conditions. More visual information is obtained if we choose the control voltage $v_{c o}$ instead of $v$ (remember the affine relation (1) between $v_{c o}$ and $v$ ), and we plot the ramp as well. In the tridimensional case, the ramp is represented by planes (see Fig. 5).

Considering that the system is $T$-periodic, and that we are only considering continuous conduction mode, the most suitable option would be to take a cylindrical space $(t, v, i) \in$ $[0, T] \times \mathbb{R} \times \mathbb{R}^{+}$, as suggested in [6]. This has the advantage that no information is lost and periodic orbits are closed trajectories, but figures become considerately complex when more than two cycles are drawn. So, when necessary, phase space and control plane plots are used instead of cylindrical ones, implicitly accepting that some information will be lost.

\section{PERIODIC ORBITS}

In this section we will look for periodic orbits of our global system. Having fixed the values of the parameters according to those of Section I, we will study for which values of the input voltage $V_{i n}$ we may have periodic orbits, and whether they are stable or not. For $r \geq 1$, we will say that $\mathcal{O}$ is an $r$-periodic orbit iff $r$ is the smallest positive integer for which

$$
\left\{\begin{array}{l}
v(r T)=v(0) \\
i(r T)=i(0)
\end{array}\right.
$$

that is, an orbit of the phase space $(v, i)$ which closes itself after $r$ cycles of the ramp. Because of (1), the above conditions are equivalent to

$$
\left\{\begin{array}{ccc}
v_{c o}(r T) & =v_{c o}(0) \\
i(r T) & =i(0) .
\end{array}\right.
$$

Since the trajectories may cross the ramp several times in a period, we may define different problems for periodic orbits. 

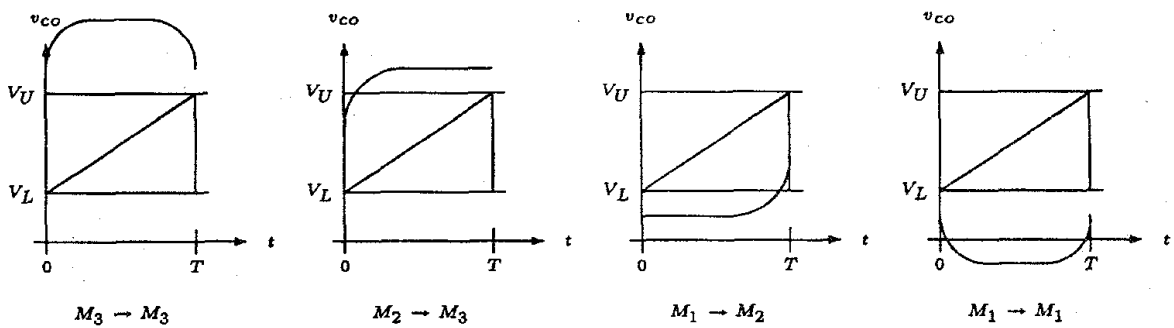

$m$ odd:

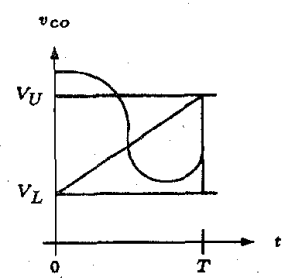

$M_{3} \rightarrow M_{2}$

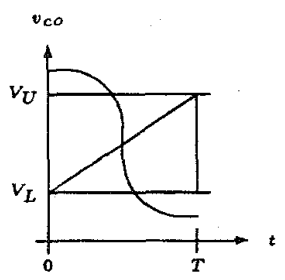

$M_{3} \rightarrow M_{1}$

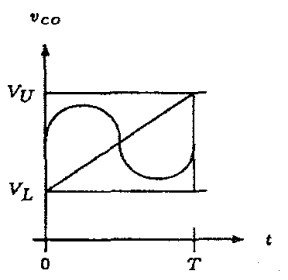

$M_{2}-M_{2}$

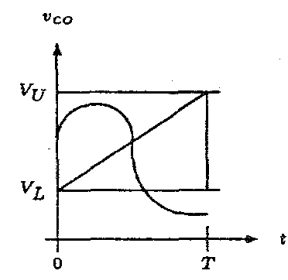

$M_{2} \rightarrow M_{1}$

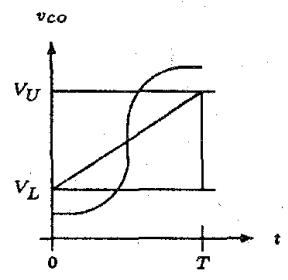

$M_{1} \rightarrow M_{3}$

Fig. 6. All the possible situations for $m$ even or odd (exemplified by $m=0$ and $m=1$ ).

Thus, if $r \geq 0$ and $m \geq 0$, we define $P(r, m)=\{r$-periodic orbits which cross the ramp at most $m$ times per period $\}$. It can be proved that, in the range of values of the parameters under consideration, (essentially $V_{i n}>V_{2} \equiv V_{U}+\frac{V_{\text {ref }}}{a}>$ $V_{1} \equiv V_{L}+\frac{V_{r e f}}{a}>0$ ), the problem of computing $P(r, 0)$ is trivial:

$$
P(r, 0)=\emptyset \quad \forall r \geq 1
$$

Indeed, if $v(r T)=v(0), i(r T)=i(0)$, and the ramp cannot be crossed, one of the following alternatives must be true

- i) $v(0)=v(r T)<V_{1}$.

- ii) $v(0)=v(r T)>V_{2}$.

for if $v(0) \in\left[V_{1}, V_{2}\right]$, the ramp would necessarily be crossed.

In case $i$ ) system 2 gives the dynamics all the time, which has no periodic orbits other than its equilibrium point $\left(V_{i n}, V_{i n} / R\right)$. But, as we are supposing $\left(V_{i n}>V_{2}>V_{1}>0\right)$, this equilibrium point cannot be considered in case i). One can prove, along the same lines, that in case ii), when the dynamics is given all the time by system 1 , we also arrive at a contradiction with $v(0)=v(r T), i(0)=i(r T)$, and so the statement is proved. Naturally, the more interesting cases $m \geq 1$ are more difficult to study. Let us consider the following partition of the range of the values of $v_{c o}$ :

$$
M_{1}=\left(-\infty, V_{L}\right), M_{2}=\left[V_{L}, V_{U}\right], M_{3}=\left(V_{U},+\infty\right) .
$$

Once the values of the parameters are fixed, we observe that if we are looking for an orbit in $P(1, m)$ verifying $v_{c o}(0) \in M_{2}$, then $m$ must be odd, while if $v_{c o}(0) \in M_{2}^{c}$, then $m$ must be even (notice that the reciprocals are also valid). Fig. 6 shows all the possibilities, and justify the above statement, which, in a more compact form, reads as follows:

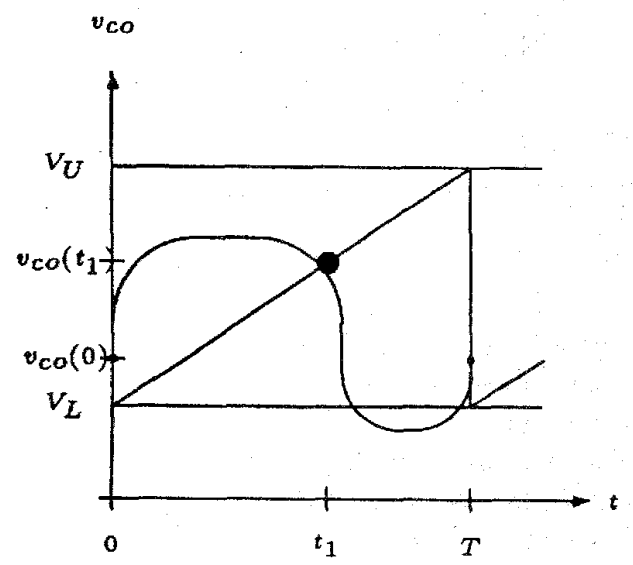

Fig. 7. Scheme of the case $P(1,1)$.

Let $\mathcal{O}$ be a 1 -periodic orbit. Then

$$
\exists m \geq 1 \quad \mathcal{O} \in P(1,2 m) \Leftrightarrow v_{c o}(0) \in M_{2}^{c} .
$$

We will study $P(1,1)$ in more detail. According to the previous note, an orbit $\mathcal{O}$ in $P(1,1)$ must start with $v_{c o}(0) \in$ $M_{2}$. Thus, it is system 1 that initially draws the orbit; at some time $t_{1}<T, v_{c o}$ crosses the ramp and system 2 enters into action till $t=T$, when one must have

$$
\left\{\begin{array}{c}
v_{c o}(T)=v_{c o}(0) \\
i(T)=i(0) .
\end{array}\right.
$$

This is graphically represented in Fig. 7.

In terms of $v(0)$, we have

$$
v_{c o}(0) \in\left[V_{L}, V_{U}\right] \Leftrightarrow v(0) \in\left[V_{1}, V_{2}\right] .
$$


The time $t_{1} \in[0, T]$ must verify

$$
\left(\begin{array}{l}
v\left(t_{1}\right) \\
i\left(t_{1}\right)
\end{array}\right)=e^{-k t_{1}}\left[I \cos \omega t_{1}+A \sin \omega t_{1}\right]\left(\begin{array}{l}
v(0) \\
i(0)
\end{array}\right)
$$

and

$$
a\left(v\left(t_{1}\right)-V_{\text {ref }}\right)=V_{L}+\frac{V_{U}-V_{L}}{T} t_{1} .
$$

From $t_{1}$ to $T$, system 2 gives the dynamics and so we must have

$$
\begin{aligned}
\left(\begin{array}{c}
v(0) \\
i(0)
\end{array}\right)= & \left(\begin{array}{c}
v(T) \\
i(T)
\end{array}\right)=\left(\begin{array}{c}
V_{i n} \\
V_{i n} / R
\end{array}\right) \\
& +e^{-k\left(T-t_{1}\right)}\left[I \cos \omega\left(T-t_{1}\right)\right. \\
& \left.+A \sin \omega\left(T-t_{1}\right)\right]\left(\begin{array}{c}
v\left(t_{1}\right)-V_{i n} \\
i\left(t_{1}\right)-V_{i n} / R
\end{array}\right)
\end{aligned}
$$

The map $N: \mathbb{R} \rightarrow M_{2 \times 2}(\mathbb{R})$ defined by

$$
N(\alpha)=e^{-k T \alpha}[I \cos \omega T \alpha+A \sin \omega T \alpha]
$$

has the following properties:

- $N(0)=\dot{I}$.

- $N(\alpha+\beta)=N(\alpha) N(\beta)$.

- $\operatorname{det}(N(\alpha))=e^{-k T \alpha}$.

In terms of $N$, if we write $t_{1}=\alpha_{1} T, \alpha_{1} \in(0,1)$ (so $\alpha_{1}$ is the duty cycle), the conditions we have imposed are equivalent to

$$
f_{1}\left(V_{i n}, \alpha_{1}\right)=0
$$

where

$$
\begin{aligned}
& f_{1}\left(V_{i n}, \alpha_{1}\right) \equiv V_{r e f}+\frac{V_{L}}{a}+\frac{V_{U}-V_{L}}{a} \alpha_{1}- \\
& (1,0) \cdot N\left(\alpha_{1}\right)\left(\begin{array}{l}
v_{0}\left(V_{i n}, \alpha_{1}\right) \\
i_{0}\left(V_{i n}, \alpha_{1}\right)
\end{array}\right)
\end{aligned}
$$

with

$$
\begin{aligned}
& \left(\begin{array}{c}
v_{0}\left(V_{i n}, \alpha_{1}\right) \\
i_{0}\left(V_{i n}, \alpha_{1}\right)
\end{array}\right)= \\
& V_{i n}[N(0)-N(1)]^{-1}\left[N(0)-N\left(1-\alpha_{1}\right)\right]\left(\begin{array}{c}
1 \\
1 / R
\end{array}\right) .
\end{aligned}
$$

Let us observe first that $N(0)-N(1)$ has an inverse, for

$$
N(0)-N(1)=I-e^{-k T}[I \cos \omega T+A \sin \omega T]
$$

and,

$$
\begin{aligned}
& \operatorname{det}\left(I-e^{-k T}[I \cos \omega T+A \sin \omega T]\right)= \\
& \left(e^{-k T}-e^{j \omega T}\right)\left(e^{-k T}-e^{-j \omega T}\right) \neq 0 .
\end{aligned}
$$

After substitution of $v_{0}\left(V_{i n}, \alpha_{1}\right)$ and $i_{0}\left(V_{i n}, \alpha_{1}\right)$ in $f_{1}\left(V_{i n}, \alpha_{1}\right)$, we have to solve (25) numerically for $V_{i n}$ and $\alpha_{1}$. Fig. 8 shows the duty cycle $\alpha_{1}$ obtained when $V_{i n}$ is varied from 12 to 50 . The initial conditions $\left(v_{0}, i_{0}\right)$, when $V_{i n}$ varies in this range, increase from $(11.7629,0.5369)$ (which

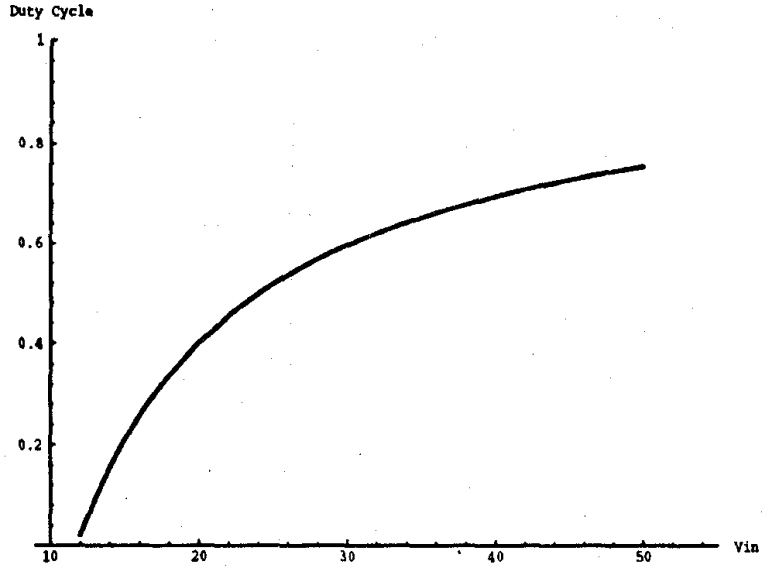

Fig. 8. Evolution of the duty cycle for the 1-periodic orbits.

corresponds to $\left.\alpha_{1}=0.02\right)$ to $(12.1576,0.6480)$ (which corresponds to $\alpha_{1}=0.7556$ ).

Notice that the conditions we imposed to obtain periodic orbits are necessary, but they are not sufficient, for it could be that $t_{1}$ is not the first time the control voltage crosses the ramp. Or, being the first, it could be that it is not the last. Thus, after computing numerically the values of $\left(v_{0}, i_{0}\right)$, we must check that the obtained trajectory is really of the desired kind. The condition $v_{0} \in\left[V_{1}, V_{2}\right]$ is guaranteed by a previous note.

Let us now study $P(2,1)$; that is, the 2-periodic orbits that cross the ramp, at most, once per period. We have seven different situations, which are represented in Fig. 9. However, we observe that, given an orbit $\mathcal{O} \in P(2,1)$, if we interchange the two periods that form $\mathcal{O}$, we get another orbit $\mathcal{O}^{\prime} \in P(2,1)$. Hence, if we interchange the two periods of an orbit of type (3), (6), or (7), we get an orbit of type (1), (4), or (2) respectively. We may say that there exists a duality between types (1) and (3), (2) and (7), and (4) and (6), while (5) is selfdual. We will restrict ourselves to the study of types (1), (2), (4), and (5), which have in common the fact that $v_{c o}(0) \in M_{1} \cup M_{2}$ and that the first time the orbits cross the ramp they do so during the first period.

We will study first type (5) which, in a sense, is the most general. If we denote by $t_{1}=\alpha_{1} T$ the first time that the control voltage crosses the ramp, we will have, with the notation previously introduced

$$
\left(\begin{array}{c}
v\left(t_{1}\right) \\
i\left(t_{1}\right)
\end{array}\right)=N\left(\alpha_{1}\right)\left(\begin{array}{c}
v_{0} \\
i_{0}
\end{array}\right)
$$

and

$$
a\left(v\left(t_{1}\right)-V_{r e f}\right)=V_{L}+\frac{V_{U}-V_{L}}{T} t_{1} .
$$

After $t_{1}$, system 2 acts until the end of the period, and we obtain

$$
\left(\begin{array}{c}
v(T) \\
i(T)
\end{array}\right)=N\left(1-\alpha_{1}\right)\left(\begin{array}{c}
v\left(t_{1}\right)-V_{i n} \\
i\left(t_{1}\right)-V_{i n} / R
\end{array}\right)+\left(\begin{array}{c}
V_{i n} \\
V_{i n} / R
\end{array}\right) .
$$


1) With $v_{c o}(0) \in M_{3}$ :

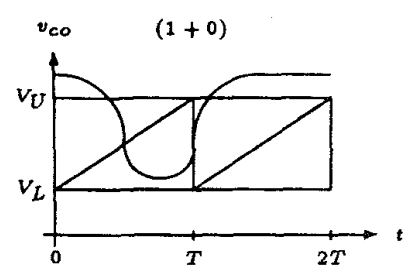

(1) $M_{3} \rightarrow M_{2} \quad M_{2} \rightarrow M_{3}$

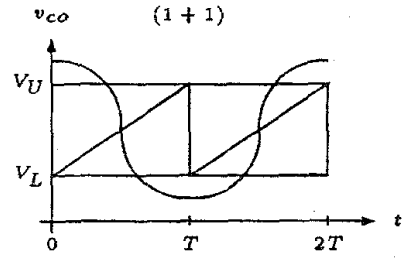

(2) $M_{3} \rightarrow M_{1} \quad M_{1} \rightarrow M_{3}$

(a)

\section{2) With $v_{c o}(0) \in M_{2}$ :}

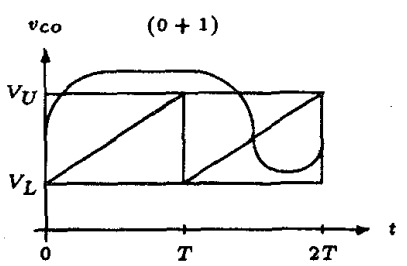

(3) $M_{2} \rightarrow M_{3} \quad M_{3} \rightarrow M_{2}$

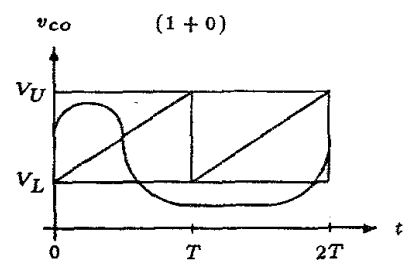

(4) $M_{2} \rightarrow M_{1} \quad M_{1} \rightarrow M_{2}$

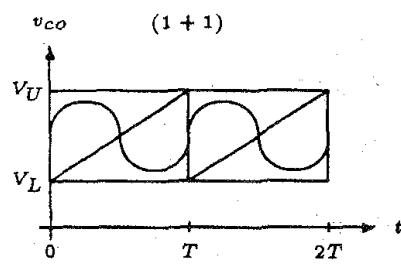

(5) $M_{2} \rightarrow M_{2} \quad M_{2} \rightarrow M_{2}$

(b)

Fig. 9. Schemes of the case $P(2,1)$.

During the second period, the ramp is crossed at $t=t_{2}=$ $\alpha_{2} T$, and we will have

$$
\left(\begin{array}{l}
v\left(t_{2}\right) \\
i\left(t_{2}\right)
\end{array}\right)=N\left(\alpha_{2}\right)\left(\begin{array}{c}
v(T) \\
i(T)
\end{array}\right)
$$

and

$$
a\left(v\left(t_{2}\right)-V_{r e f}\right)=V_{L}+\frac{V_{U}-V_{L}}{T} t_{2} .
$$

During the second part of the second period the dynamics is given again by system 2 and

$$
\left(\begin{array}{c}
v(2 T) \\
i(2 T)
\end{array}\right)=N\left(1-\alpha_{2}\right)\left(\begin{array}{c}
v\left(t_{2}\right)-V_{i n} \\
i\left(t_{2}\right)-V_{i n} / R
\end{array}\right)+\left(\begin{array}{c}
V_{i n} \\
V_{i n} / R
\end{array}\right) .
$$

Finally, to get a 2 -periodic orbit, we must impose

$$
\left(\begin{array}{c}
v(2 T) \\
i(2 T)
\end{array}\right)=\left(\begin{array}{c}
v_{0} \\
i_{0}
\end{array}\right)
$$

In a similar way, as in the case of $P(1,1)$, we obtain

$$
\begin{aligned}
& \left(\begin{array}{c}
v_{0} \\
i_{0}
\end{array}\right)=V_{i n}[N(0)-N(2)]^{-1}\left[N(0)-N\left(1-\alpha_{2}\right)\right. \\
& \left.+N(1)-N\left(2-\alpha_{1}\right)\right]\left(\begin{array}{c}
1 \\
1 / R
\end{array}\right)
\end{aligned}
$$

with additional conditions (29) and (32).

If we substitute the values of $v\left(t_{1}\right)$ and $v\left(t_{2}\right)$ in terms of $v_{0}$ and $i_{0},(29)$ and (32) are equivalent to

$$
\left\{\begin{array}{l}
f_{1}\left(V_{i n}, \alpha_{1}, \alpha_{2}\right)=0 \\
f_{2}\left(V_{i n}, \alpha_{1}, \alpha_{2}\right)=0
\end{array}\right.
$$

where

$$
\begin{aligned}
& f_{1}\left(V_{i n}, \alpha_{1}, \alpha_{2}\right)=V_{r e f}+\frac{V_{L}}{a}+\frac{V_{U}-V_{L}}{a} \alpha_{1}- \\
& (1,0) \cdot N\left(\alpha_{1}\right)\left(\begin{array}{l}
v_{0}\left(V_{i n}, \alpha_{1}, \alpha_{2}\right) \\
i_{0}\left(V_{i n}, \alpha_{1}, \alpha_{2}\right)
\end{array}\right)
\end{aligned}
$$

and

$$
\begin{aligned}
& f_{2}\left(V_{i n}, \alpha_{1}, \alpha_{2}\right)= \\
& V_{\text {ref }}+\frac{V_{L}}{a}+\frac{V_{U}-V_{L}}{a} \alpha_{2}- \\
& (1,0) \cdot\left[N\left(1+\alpha_{2}\right)\left(\begin{array}{l}
v_{0}\left(V_{i n}, \alpha_{1}, \alpha_{2}\right) \\
i_{0}\left(V_{i n}, \alpha_{1}, \alpha_{2}\right)
\end{array}\right)+\right. \\
& \left.V_{i n}\left(N\left(\alpha_{2}\right)-N\left(1-\alpha_{1}+\alpha_{2}\right)\right)\left(\begin{array}{c}
1 \\
1 / R
\end{array}\right)\right] .
\end{aligned}
$$

Before going on, let us point out that in cases (1), (2), and (4) we obtain similar expressions. For instance, in case (1), the expression for $\left(v_{0}, i_{0}\right)$ is the same as in case (5) with $\alpha_{2}=1$ (see Fig. 9). Now, condition $f_{2}\left(V_{i n}, \alpha_{1}, \alpha_{2}\right)=0$ must not be imposed and we obtain

$$
\left(\begin{array}{c}
v_{0} \\
i_{0}
\end{array}\right)=V_{i n}[N(0)-N(2)]^{-1}\left[N(1)-N\left(2-\alpha_{1}\right)\right]\left(\begin{array}{c}
1 \\
1 / R
\end{array}\right)
$$

with (25).

Case (4) can also be studied in a similar way, but from Fig. 9 we see that it is the same as case (5) with $\alpha_{2}=0$. Since there is no second crossing with the ramp we do not 


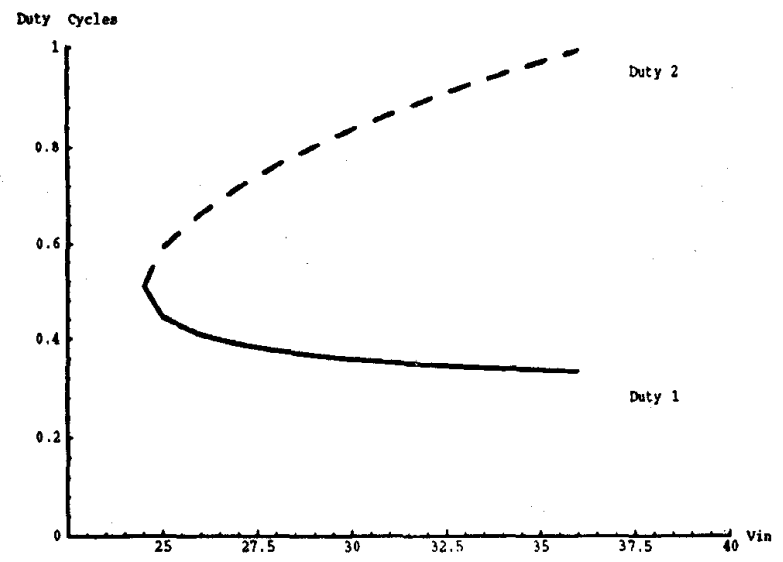

Fig. 10. Evolution of the duty cycles for the 2-periodic orbits.

have to impose $f_{2}\left(V_{i n}, \alpha_{1}, \alpha_{2}\right)=0$, and we obtain

$$
\left(\begin{array}{c}
v_{0} \\
i_{0}
\end{array}\right)=V_{i n}[N(0)-N(2)]^{-1}\left[N(0)-N\left(2-\alpha_{1}\right)\right]\left(\begin{array}{c}
1 \\
1 / R
\end{array}\right)
$$

with condition (25).

Case (2) is different from the previous ones because the second cycle starts with system 2 and ends with system 1. Similarly to what we did in case (5), we get

$$
\begin{aligned}
& \left(\begin{array}{c}
v_{0} \\
i_{0}
\end{array}\right)= \\
& V_{i n}[N(0)-N(2)]^{-1}\left[N\left(1-\alpha_{2}\right)-N\left(2-\alpha_{1}\right)\right]\left(\begin{array}{c}
1 \\
1 / R
\end{array}\right)
\end{aligned}
$$

with the additional conditions (29), (32) and $v_{c o}(0) \in M_{3}$.

In all the cases, three comments should be made before starting the numerical computations. First, when computing the duty cycles for a given value $V_{i n}$, if a pair $\left(\alpha_{1}, \alpha_{2}\right)$ is obtained, then $\left(\alpha_{2}, \alpha_{1}\right)$ must also be a solution (interchanging the two periods of the orbit), provided that $\left(v_{0}, i_{0}\right)$ and $(v(T), i(T))$ are also interchanged. Second, solutions of the form $\alpha_{1}=\alpha_{2}$ must exist which correspond to orbits $\mathcal{O} \in P(1,1) \subseteq P(1,2)$. Third, we must remember that the conditions imposed are necessary and not sufficient, for the same reason as in case $P(1,1)$. So, once $\left(v_{0}, i_{0}\right)$ have been obtained, we must draw the full orbit over the two periods to ensure that the number of crossings in the ramp is correct. Numerical computations in case (5) are shown in Fig. 10.

From $V_{\text {in }}$ near 24.516 onwards we start obtaining duty cycles $\left(\alpha_{1}, \alpha_{2}\right) \in[0,1] \times[0,1],\left(\right.$ for $V_{i n}=24.516, \alpha_{1} \approx$ $\alpha_{2} \approx 0.51$ ), till we arrive to $V_{\text {in }}=36$, when $\alpha_{2}=1$ and $\alpha_{1}=0.3340$. The initial condition $v_{0}$ increases from 12.0277 to 12.2669 while $i_{0}$ decreases from 0.6081 to 0.4718 . Fig. 11 shows the 2-periodic orbit corresponding to $V_{\text {in }}=25$ in the phase space $(v, i)$ and Fig. 12 shows the same orbit in $\left(t, v_{c o}\right)$-axes with the ramp.

Numerical computations are not as easy as in the case of $P(1,1)$. For fixed $V_{i n}$, we should find $\left(\alpha_{1}, \alpha_{2}\right) \in[0,1] \times[0,1]$

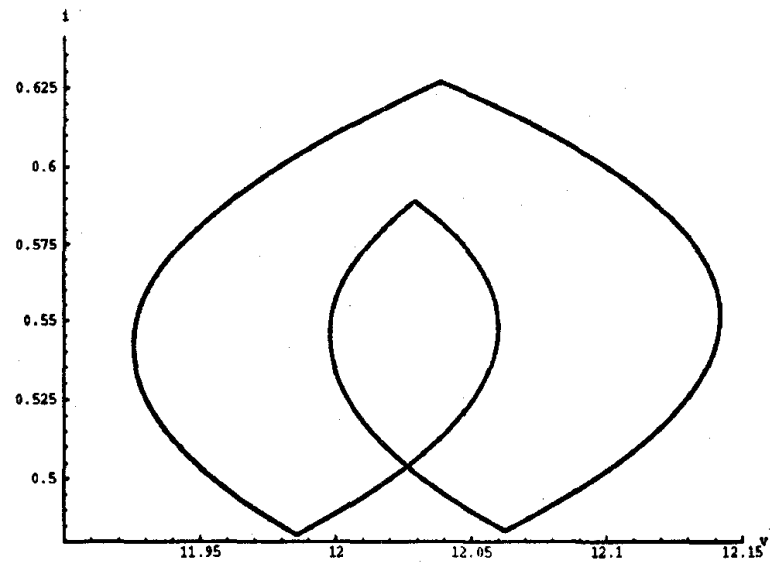

Fig. 11. Two-periodic orbit in the phase space $(v, i)$ corresponding to $V_{i n}=25$.

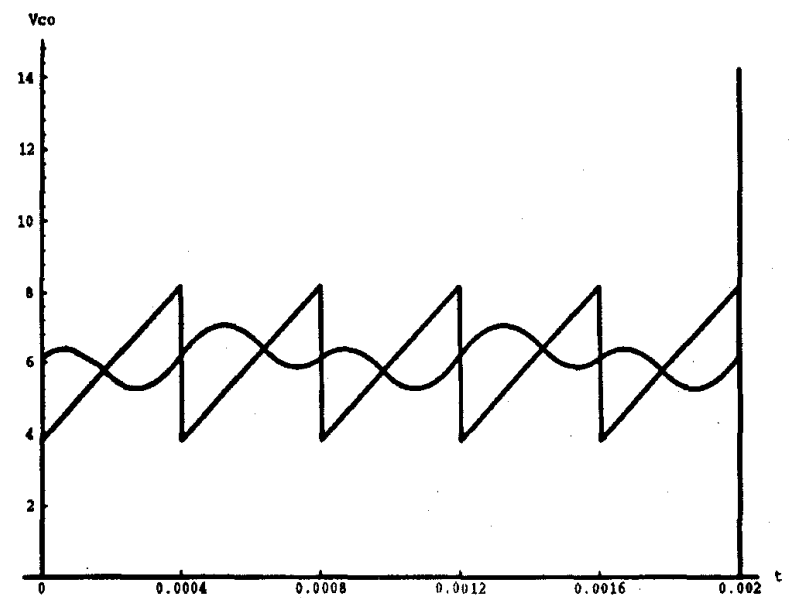

Fig. 12. Two-periodic orbit in the $\left(t, v_{c o}\right)$-space corresponding to $V_{\text {in }}=25$.

such that

$$
\left\{\begin{array}{l}
f_{1}\left(V_{i n}, \alpha_{1}, \alpha_{2}\right)=0 \\
f_{2}\left(V_{i n}, \alpha_{1}, \alpha_{2}\right)=0
\end{array}\right.
$$

With $V_{i n}$ fixed, if the surfaces $z=f_{1}\left(V_{i n}, \alpha_{1}, \alpha_{2}\right)$, and $z=f_{2}\left(V_{i n}, \alpha_{1}, \alpha_{2}\right)$ are graphically represented, together with the plane $z=0$ for $\left(\alpha_{1}, \alpha_{2}\right) \in[0,1] \times[0,1]$, it turns out that $f_{1}$ and $f_{2}$ are too close to see how many times they intersect. It is better to represent instead $z=f_{1}\left(V_{i n}, \alpha_{1}, \alpha_{2}\right)$ $f_{2}\left(V_{i n}, \alpha_{1}, \alpha_{2}\right), z=f_{1}\left(V_{i n}, \alpha_{1}, \alpha_{2}\right)$ and $z=0$, which have the same intersection as the previous set. For values above $V_{i n}=24.516$ three points of intersection are observed. One of them is of the form $(\alpha, \alpha)$, corresponding to an orbit of $P(1,1)$ and the other two are of the form $\left(\alpha_{1}, \alpha_{2}\right)$ and $\left(\alpha_{2}, \alpha_{1}\right)$, as we anticipated.

In the general case of $r$-periodic orbits, we have only studied the situation when exactly one crossing per cycle occurs and 
$v_{c o} \in M_{2}$. In this case we get

$$
\begin{aligned}
& \left(\begin{array}{l}
v_{0}\left(V_{i n}, \alpha_{1}, \ldots, \alpha_{r}\right) \\
i_{0}\left(V_{i n}, \alpha_{1}, \ldots, \alpha_{r}\right)
\end{array}\right)=V_{i n}[N(0)-N(r)]^{-1}\left[\sum_{j=0}^{r-1}(N(j)\right. \\
& \left.\left.-N\left(j+1-\alpha_{r+1-j}\right)\right)\right]\left(\begin{array}{c}
1 \\
1 / R
\end{array}\right)
\end{aligned}
$$

with the additional conditions

$$
\left\{\begin{array}{c}
f_{1}\left(V_{i n}, \alpha_{1}, \ldots, \alpha_{r}\right)=0 \\
\ldots \\
f_{r}\left(V_{i n}, \alpha_{1}, \ldots, \alpha_{r}\right)=0
\end{array}\right.
$$

where

$$
\begin{aligned}
& f_{j}\left(V_{i n}, \alpha_{1}, \ldots, \alpha_{r}\right)=V_{r e f}+\frac{V_{L}}{a}+\frac{V_{U}-V_{L}}{a} \alpha_{j} \\
& -(1,0) \cdot N\left(\alpha_{j}\right)\left(\begin{array}{c}
v_{j-1} \\
i_{j-1}
\end{array}\right)
\end{aligned}
$$

with

$$
\left(\begin{array}{c}
v_{j} \\
i_{j}
\end{array}\right)=N(1)\left(\begin{array}{c}
v_{j-1} \\
i_{j-1}
\end{array}\right)+V_{i n}\left[N(0)-N\left(1-\alpha_{j}\right)\right]\left(\begin{array}{c}
1 \\
1 / R
\end{array}\right) .
$$

Thus, in this case, the problem of finding the periodic orbits is reduced to numerically solving the equations

$$
\left\{\begin{array}{c}
f_{1}\left(V_{i n}, \alpha_{1}, \ldots, \alpha_{r}\right)=0 \\
\ldots \\
f_{r}\left(V_{i n}, \alpha_{1}, \ldots, \alpha_{r}\right)=0
\end{array}\right.
$$

in the region $\left(\alpha_{1}, \ldots, \alpha_{r}\right) \in[0,1]^{r}$ and then check that $v_{c o}(0) \in M_{2}$.

\section{STABILITY}

We now propose to study the stability of the 1 and 2 periodic orbits that we found in the previous section. Given a periodic orbit, we will say that it is asymptotically stable if, given initial conditions $\left(v_{0}, i_{0}\right)$ sufficiently close to the periodic orbit, the trajectory in the phase space from $\left(v_{0}, i_{0}\right)$ asymptotically approaches that of the periodic orbit. The characteristic multipliers test is used to decide whether a periodic orbit is stable or not. It is known [6], [7], that if the norms of all the characteristic multipliers of a periodic orbit are less than 1 , then the orbit is stable, while one characteristic multiplier with norm greater than 1 suffices to render the periodic orbit unstable.

We have computed the characteristic multipliers as follows. We constructed the Poincaré maps, which are given by

$$
\begin{aligned}
& P: \mathbb{R}^{2} \longrightarrow \mathbb{R}^{2} \\
& \left(v_{0}, i_{0}\right) \longrightarrow P\left(v_{0}, i_{0}\right)=(v(T), i(T))
\end{aligned}
$$

for a 1-periodic orbit and

$$
\begin{aligned}
& P: \mathbb{R}^{2} \longrightarrow \mathbb{R}^{2} \\
& \left(v_{0}, i_{0}\right) \longrightarrow P\left(v_{0}, i_{0}\right)=(v(2 T), i(2 T))
\end{aligned}
$$

for a 2-periodic orbit.

These maps give us the state of the system after 1 or 2 periods, respectively, starting from initial conditions $\left(v_{0}, i_{0}\right)$.

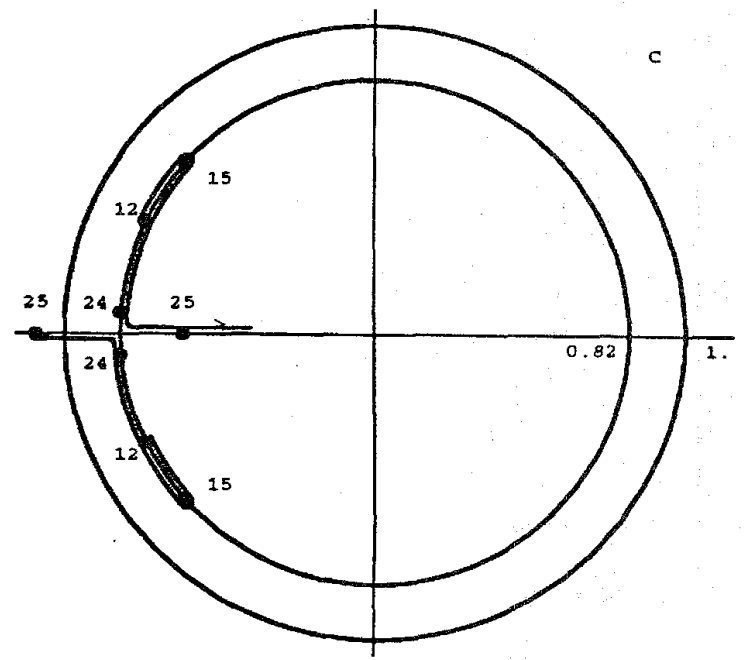

Fig. 13. Evolution of the characteristic multipliers for the 1-periodic orbits as a function of $V_{i n}$ (the numbers are the values of $V_{i n}$ in the range [12,25]. Outer radius is 1 ; inner radius is 0.82 ).

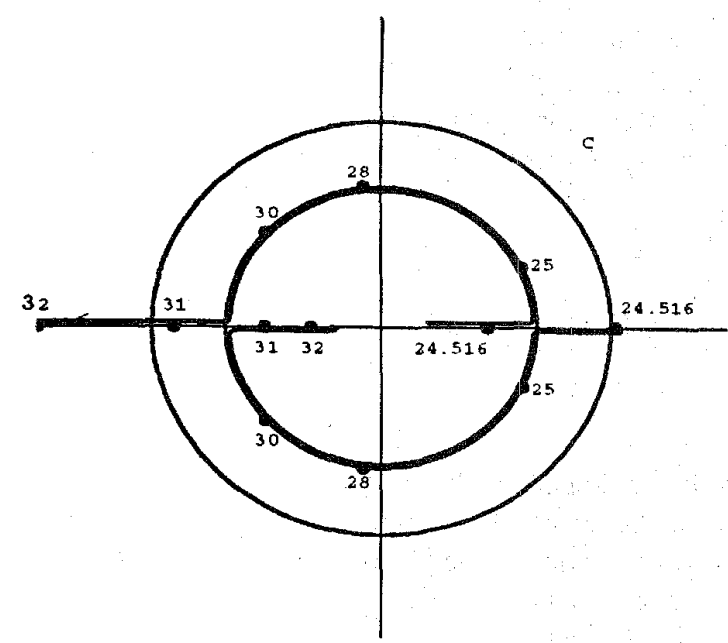

Fig. 14. Evolution of the characteristic multipliers for the 2-periodic orbits as a function of $V_{i n}$ (the numbers are the values of $V_{i n}$ in the range [24.516,32]. Outer radius is 1 ; inner radius is 0.679 ).

The characteristic multipliers are then the eigenvalues of $D P\left(\bar{v}_{0}, \bar{i}_{0}\right)$, where $\left(\bar{v}_{0}, \bar{i}_{0}\right)$ are the initial conditions corresponding to a given periodic orbit [6], [7]. We have computed numerically $D P\left(v_{0}, i_{0}\right)$ using central differences and Richardson extrapolation [8] and the results are graphically shown in Fig. 13 and Fig. 14. In Fig. 13 we draw in the complex plane the evolution of the characteristic multipliers for the 1-periodic orbits when $V_{\text {in }}$ sweeps the range from 12 to 25. We observe first that the characteristic multipliers are complex conjugates that move on a circle of radius $\approx 0.82$, and so the orbit is asymptotically stable. Near $V_{i n}=24$, the characteristic multipliers become both real, and when $V_{i n}$ reaches a certain value between $V_{i n}=24$ and $V_{i n}=25$, one of the characteristic multipliers has norm greater than 1 , and 


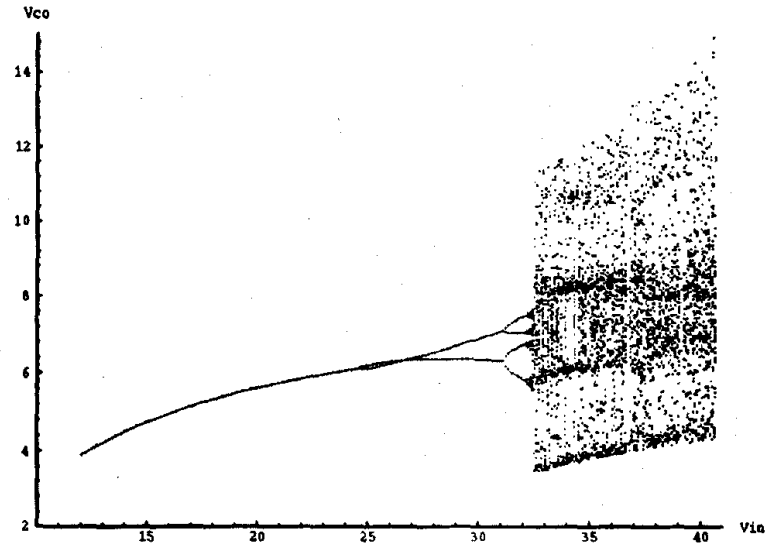

Fig. 15. Bifurcation diagram of $v_{c o}$ taking $V_{i n}$ as bifurcation parameter.

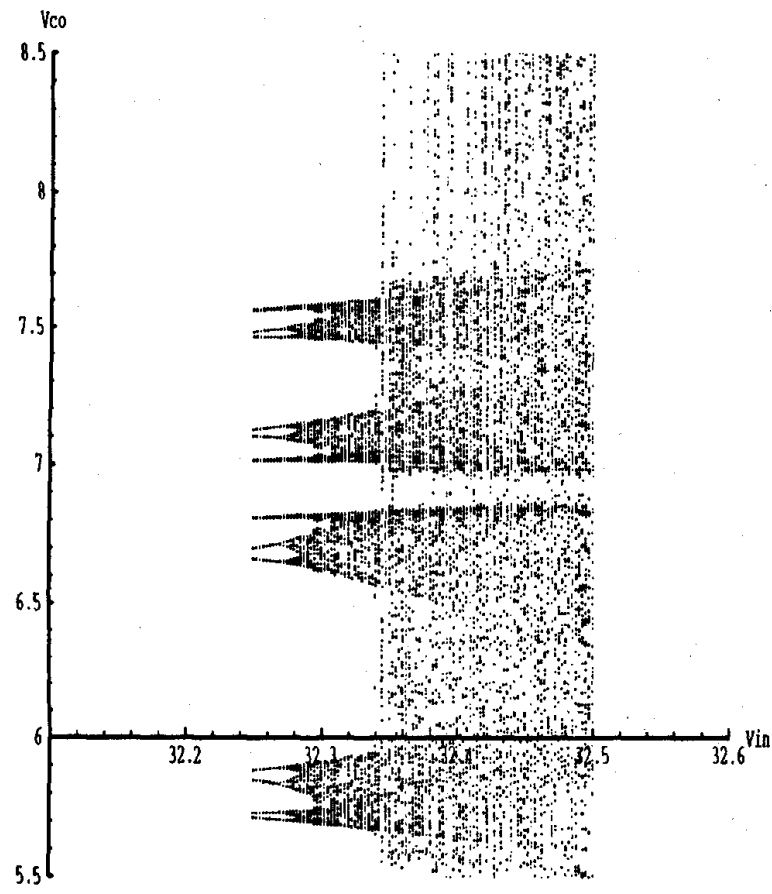

Fig. 16. Bifurcation diagram of $v_{c o}$ taking $V_{i n}$ as bifurcation parameter. First apparently chaotic zone.

so the periodic orbit becomes unstable. After $V_{i n}=25$, it remains unstable.

Fig. 14 shows the same kind of diagram in the case of 2periodic orbits. Near $V_{\text {in }}=25$ both multipliers enter the circle of radius 1 , yielding stable orbits. Next, they move on the circle of radius 0.679 till $V_{i n}=31$ is reached. After $V_{i n}=31$, one of the multipliers goes out of the unit circle and the stability is lost and not recovered. In order to obtain a clearer understanding of what is going on, one may construct a bifurcation diagram of $v_{c o}$ taking $V_{i n}$ as the bifurcation parameter [2]. This has been done in Fig. 15, which shows a first bifurcation for $V_{i n}=24.516$ and a second one for $V_{i n}=31.455$.

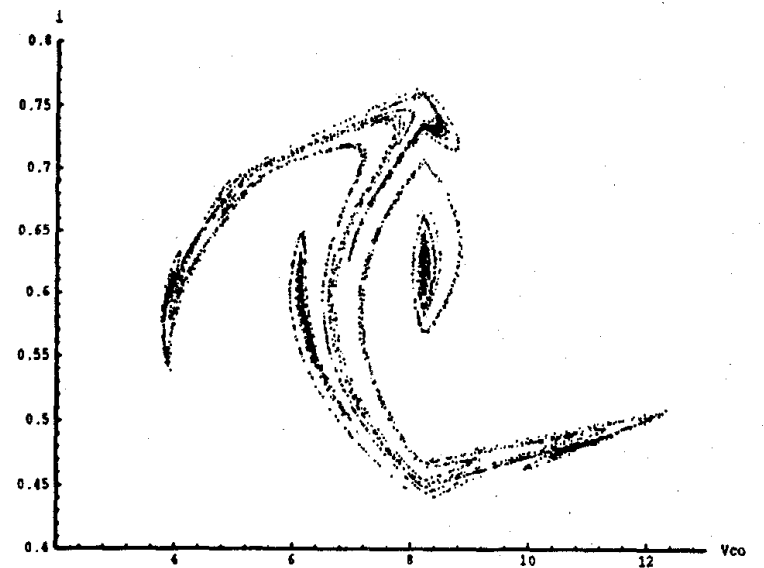

Fig. 17. Strange attractor for $V_{i n}=35$ (numerical simulation of 4000 iterations of the Poincaré map).

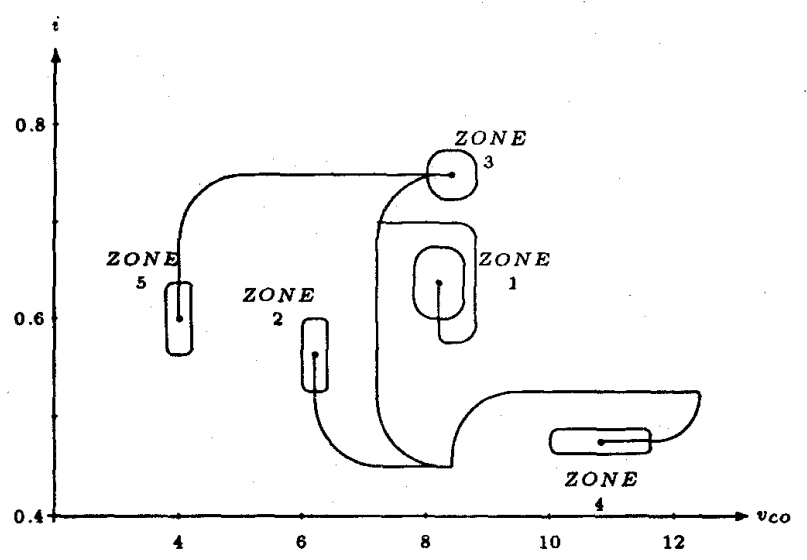

Fig. 18. Scheme of the attractor for $V_{i n}=35$.

Then, for higher values of $V_{i n}$, a cascade of perioddoublings follows, till an apparently chaotic zone is reached. This zone is shown in detail in Fig. 16. A careful observation of the numerical data allows some $2^{k}$-periods to be identified. From $V_{i n}=32.2775$, which corresponds to a $2^{6}$-period, if we successively increase the input voltage by an amount of 0.0025 , periods $3 \cdot 2^{5}\left(V_{i n}=32.28\right), 3 \cdot 2^{4}\left(V_{i n}=32.2825\right)$ and $7 \cdot 2^{3}\left(V_{\text {in }}=32.29\right)$ seem to be recognized.

Let us consider the Poincaré map for one period

$$
\begin{aligned}
P: \mathbb{R}^{2} & \longrightarrow \mathbb{R}^{2} \\
\left(v_{c o}(0), i(0)\right) & \longrightarrow P\left(v_{c o}(0), i(0)\right)=\left(v_{c o}(T), i(T)\right) .
\end{aligned}
$$

If we start with a given $x_{0}=\left(v_{\mathrm{co}}(0), i(0)\right)$ and, iterating $P$, we compute $\left\{x_{0}, P\left(x_{0}\right), P^{2}\left(x_{0}\right), P^{3}\left(x_{0}\right), \ldots\right\}$, we get, depending on the values of $V_{i n}$, very different behavior. When $V_{i n}<24.516$ the sequence $\left\{P^{n}\left(x_{0}\right)\right\}_{n}$ converges to $\left(\bar{v}_{c o}, \bar{i}\right)$, which are the values of the initial conditions corresponding to the stable 1 -periodic orbits. In the range $V_{\text {in }} \in[24.516,31.455]$, the sequence has accumulation points $\bar{x}_{1}$ and $\bar{x}_{2}$, where $\bar{x}_{1}$ is the initial condition that gives rise to a 2 -periodic orbit and $\bar{x}_{2}=P\left(\bar{x}_{1}\right)$. When, after a cascade of 

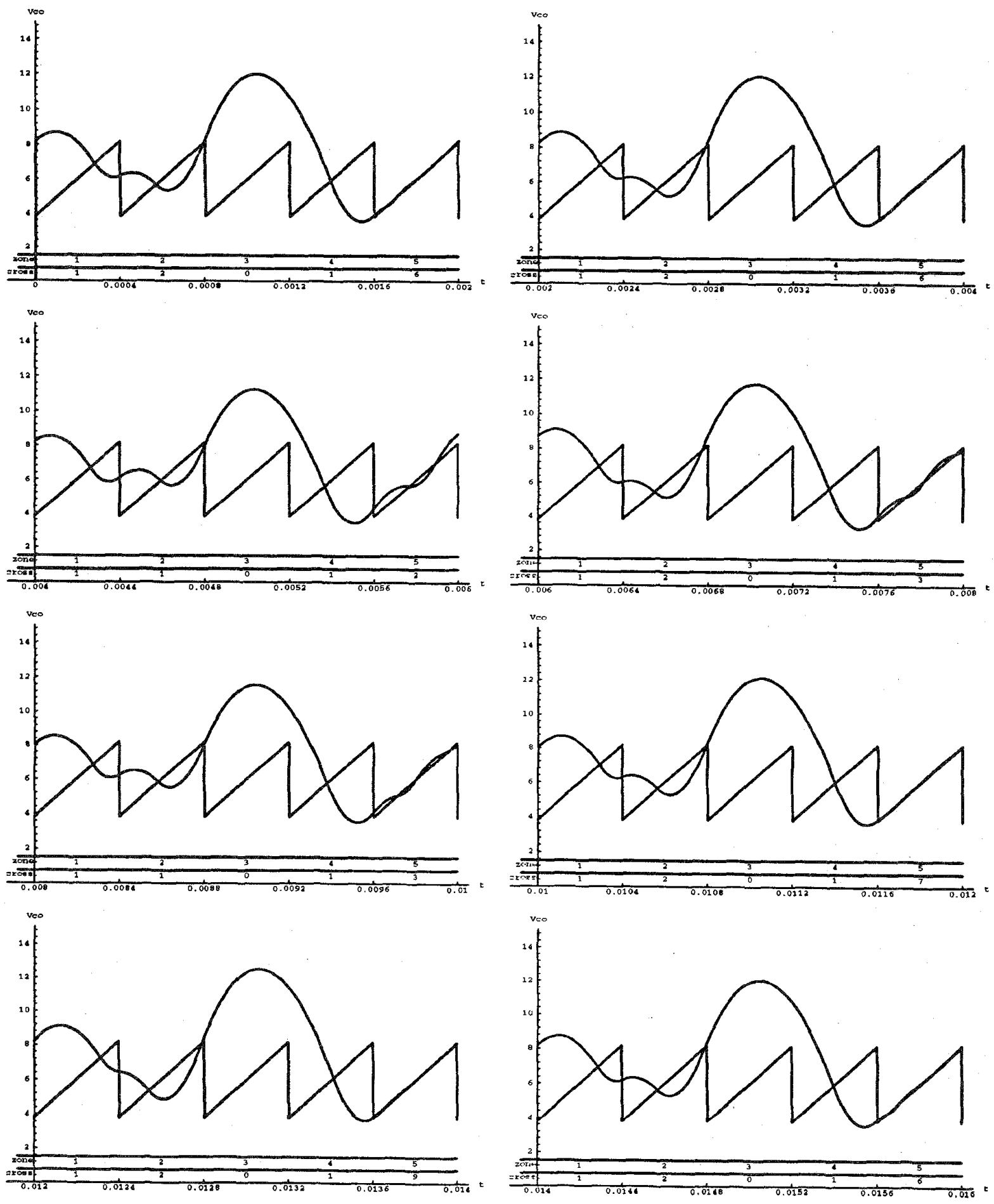

Fig. 19. 40 cycles, displayed in groups of 5 , with initial conditions near the strange attractor.

period doublings, one reaches the chaotic zone, for instance $V_{i n}=35$, we obtain a graph like that of Fig. 17 .

Both in [1] and [2] this possibly strange attractor was experimentally obtained, and in [2] was also numerically computed, obtaining only part of the experimental simulation. Notice that Fig. 17 shows exactly the other part of the experimental simulation in [1].

If we observe in detail the behavior of the sequence when the strange attractor has been reached, we may point out several characteristics of the orbits in the plane $\left(v_{c o}, i\right)$. First 
of all, the orbit visits five zones on a regular basis. The last one corresponds to multiple crossings in the ramp per period, while for the other four at most two crossings appear. We have (see also Fig. 18)

zone 1: 1 crossing.

zone $2: 1$ or 2 crossings.

zone 3: 0 or 1 crossing.

zone 4: 1 crossing.

zone 5: multiple crossings ( 0 to $\infty$ ).

Starting with initial conditions near the strange attractor, 40 cycles are plotted in Fig. 19 in the $\left(t, v_{c o}\right)$-representation, and the numbers of the zone and crossings in the ramp are also given. A certain degree of periodicity (every 5 periods) can be observed, together with the five zones previously described. In the next section we will study a careful delimitation of the five zones and the number of crossings in each one in greater detail.

\section{THE Number of Crossings}

Multiple crossings per period in the ramp pose serious difficulties to the detailed study of the dynamics of the system. What we have done is to try to delimit the zone of multiple crossings depending on the initial conditions we take. In general, one observes that the number of crossings increases with $V_{i n}$. Taking $V_{i n}=35$, inside the proposed chaotic zone where the strange attractor appears, Fig. 20 shows, for each $\left(v_{0}, i_{0}\right)$, the number of crossings in the ramp when the system evolves from the initial conditions $\left(v_{0}, i_{0}\right)$ to the end of one cycle $(v(T), i(T))$. The black zone corresponds to zero crossings, the gray zone to one crossing, the light gray zone to two crossings, and finally, the white zone to three or more crossings. Thus, the zone that corresponds to multiple crossings is bounded in the phase space. Fig. 21 shows the white zone and its neighborhood in more detail, where the concatenation of the regions of multiple crossings is seen.

The central point corresponds to the initial conditions $\left(v_{0}, i_{0}\right)$ necessary to $v_{c o}$ when it attempts to follow $v_{\text {ramp }}$ at the beginning of the ramp. So, we must have

$$
v_{c o}(0)=V_{L}, \quad \frac{d v_{c o}}{d t}(0)=\frac{V_{U}-V_{L}}{T} .
$$

Substitution of these conditions in the equations of the system gives

$$
\begin{array}{r}
v_{0}=V_{\text {ref }}+\frac{V_{L}}{a} \approx 11.75238095 \\
i_{0}=\frac{v_{0}}{R}+\frac{C\left(V_{U}-V_{L}\right)}{a T} \approx 0.595746753
\end{array}
$$

which corresponds to the initial conditions necessary to get theoretically $\infty$ crossings in the ramp. Notice from (52) that this central point depends on all the parameters of the circuit except $L$ and the input voltage $V_{i n}$. In particular, in all our simulations, as $V_{i n}$ is the only parameter varied, the central point remains the same.

Looking at Fig. 20 and Fig. 21, we may define a partition of the phase space $(v, i)$ into regions $R_{0}, R_{1}, R_{2}, \ldots$, where $R_{n}=\left\{\right.$ initial conditions $\left(v_{0}, i_{0}\right)$ that give $n$ crossings in one period of the ramp $\}$. All the regions have two bounded and connected components, except for $R_{0}$ and $R_{1}$ that are

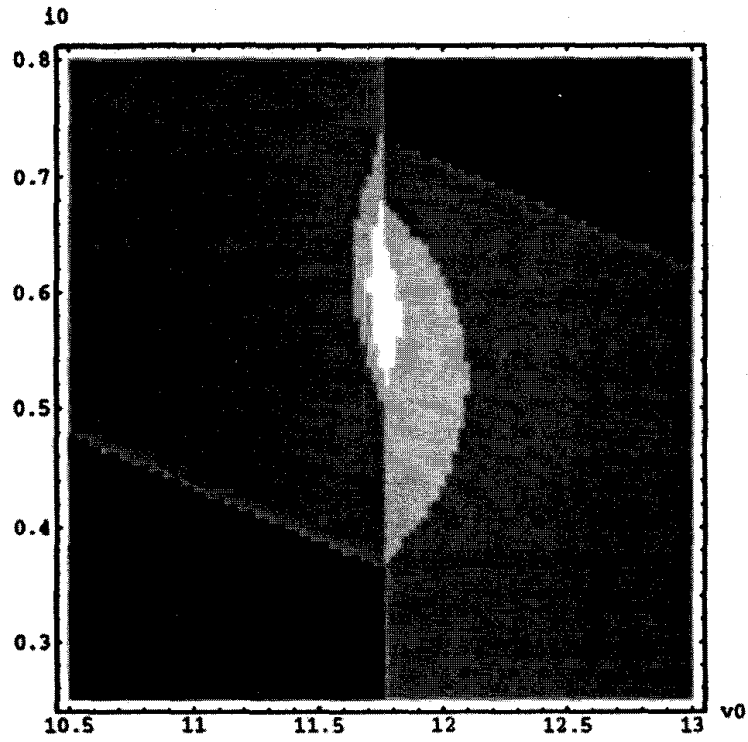

Fig. 20. Number of crossings in the ramp $\left(V_{i n}=35\right)$.

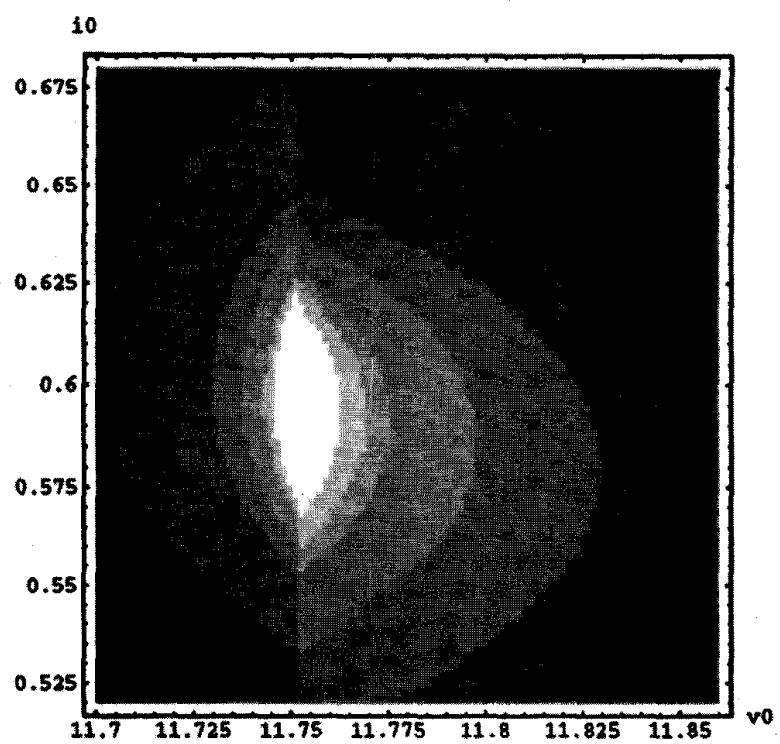

Fig. 21. Number of crossings in the ramp. Detail of the central zone $\left(V_{i n}=35\right)$.

unbounded. Moreover, $R_{0}$ has border only with $R_{1}$, and, for $n \geq 1, R_{n}$ has border only with $R_{n-1}$ and $R_{n+1}$. We have computed the images of the regions $R_{n}$ when we apply to them the Poincaré map; that is, the Poincaré map restricted to $R_{n}$

$$
\begin{aligned}
& P: R_{n} \longrightarrow \mathbb{R}^{2} \\
& \left(v_{0}, i_{0}\right) \longrightarrow(v(T), i(T)) .
\end{aligned}
$$

Fig. 22 shows part of the images of $R_{0}$ and $R_{1}$ (they are unbounded) and the whole images of $R_{2}$ and $\cup_{n \geq 3} R_{n}$. The images correspond to the dotted zones. The regions corresponding to zero crossings, one crossing and two or more crossings have also been displayed schematically.

The first plot of Fig. 22 tells us that the image of $R_{0}$ is divided into zones of zero crossings, $R_{0}$, and one crossing, 


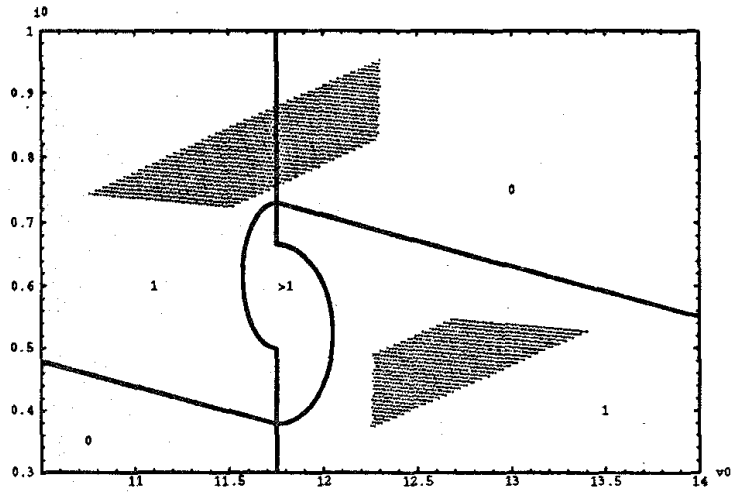

Partial plot of $P\left(R_{0}\right)$

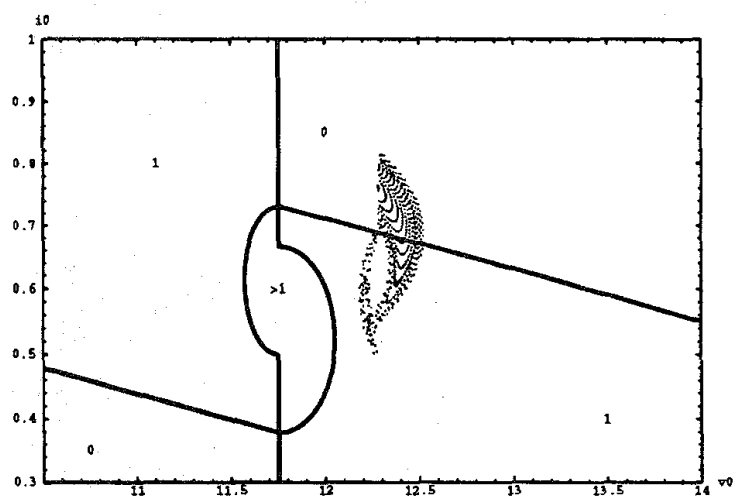

Plot of $P\left(R_{2}\right)$

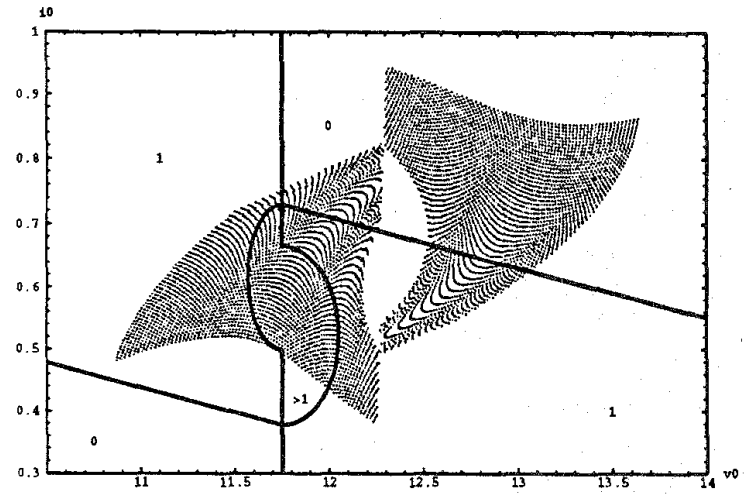

Partial plot of $P\left(R_{1}\right)$

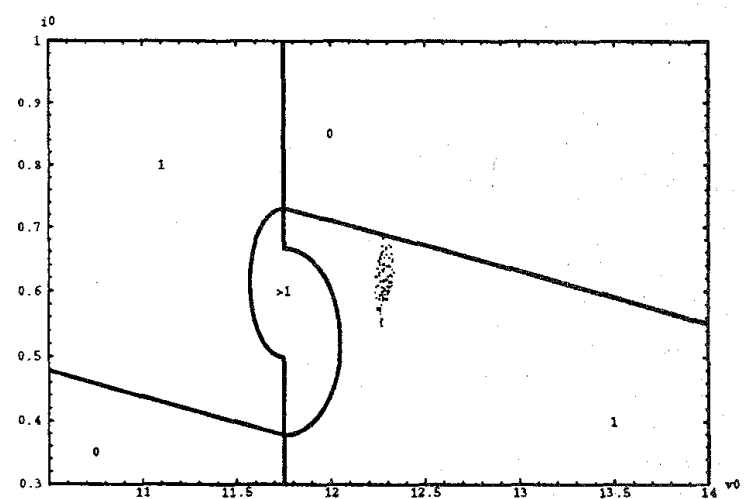

Plot of $P\left(R_{3} \cup R_{4} \cup \ldots\right)$

Fig. 22. Images of the regions by the Poincare map $\left(V_{i n}=35\right)$.

$R_{1}$. That is, the cycle following a cycle with zero crossings can have either zero or one crossing. The second plot indicates that the cycle following a cycle with one crossing can have any number of crossings, for the image of $R_{1}$ has no empty intersection with the zone of multiple crossings, and it includes the central point. The third plot shows that after a cycle with two crossings, only a cycle with one or zero crossings may follow, and finally, one crossing is expected after a cycle with three or more crossings, as is shown by the fourth plot. This is in agreement with the movement between the zones of the attractor that we discussed in the previous section.

Finally, we must point out that the image of the region in the phase space $[11.70,11.86] \times[0.52,0.68]$ by the Poincare map, shows a pronounced winding (see Fig. 23). This motivates the search for a horseshoe in the Poincaré map [9], whose existence would confirm the chaotic behavior that we are looking for, in the range of values of $V_{i n}$ near 35 .

\section{CONClusion}

Some analytical results have been obtained when searching for periodic orbits. Concretely, in the case of crossing the ramp voltage once per cycle, we found a recurrent expression for the

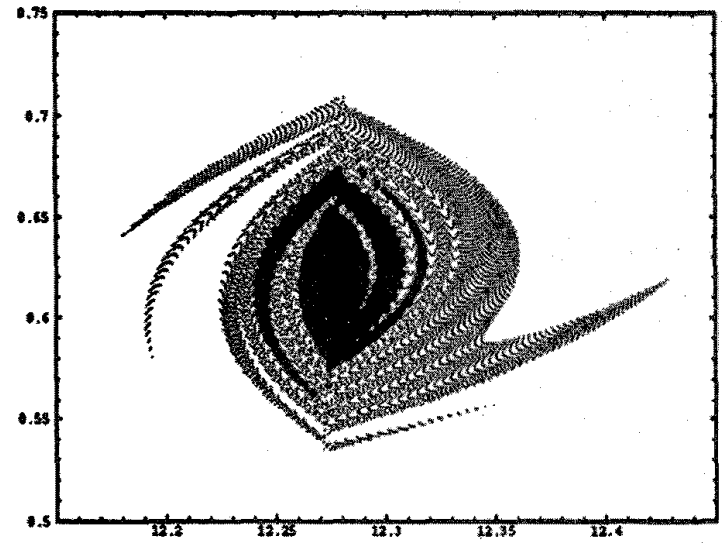

Fig. 23. Image of the region $[11.70,11.86] \times[0.52,0.68]$ by the Poincaré map $\left(V_{i n}=35\right)$. Black zone corresponds to more than three crossings.

initial conditions giving $r$-periodicity. Owing to the inherent complexity, one must finally resort to numerical methods to obtain quantitative results. They also had to be applied to determine the stability of periodic orbits. We have built a small library of programs, which simulate the circuit and display the 
results in a variety of graphs, showing skipped cycles, multiple pulsing, subharmonics and a period-doubling route to chaos.

Although all the simulations in this paper have been done with a concrete set of values of the parameters, the input voltage being the only parameter varied, similar results with the same properties are obtained when the values of the parameters are taken in a wide region containing those of the concrete set.

In the chaotic regime a strange attractor is found. The analysis of the number of crossings in the ramp reveals to be a helpful tool for following the orbits when they reach the attractor, and suggests that a horseshoe mechanism in the Poincaré map could exist. Our investigation into chaos in the buck converter continues in this direction, where not only simulation but analytical justifications of such a mechanism would be interesting.

Our goal is to gain sufficient understanding that converters could be designed with reliability and predictability in their performance, when operating under instability or even chaotically.

\section{ACKNOWLEDGMENT}

The authors wish to acknowledge the anonymous reviewers who brought important suggestions to improve the original manuscript. We also wish to thank Dr. L. Martínez for his valuable comments on a draft of this paper.

\section{REFERENCES}

[1] J. H. B. Deane and D. C. Hamill, "Analysis, simulation and experimental study of chaos in the buck converter," in IEEE Power Electronics Specialists Conf., June 1990, pp. 491-498.

[2] D. C. Hamill, J. H. B. Deane, and D. J. Jefferies, "Modeling of chaotic DC-DC converters by iterated nonlinear mappings," IEEE Trans. Power Electron., vol. 7. pp. 25-36. Jan. 1992.

[3] J. R. Wood, "Chaos: A real phenomenon in power electronics," in 1989 IEEE Applied Power Electron. Conf. Rec., pp. 115-124.

[4] G. Salut, J. C. Marpinard, and M. Valentin, "Large signal feedback control for power switching conversion," in 1985 IEEE PESC Rec., pp. $741-750$.
[5] J. H. B. Deane and D. C. Hamill, "Chaotic behavior in current-mode controlled dc-dc convertor," Electron. Lett., vol. 27, no. 13. pp. 1172 1173, June 1991

[6] T. S. Parker and L. O. Chua, Practical Numerical Algorithms for Chaotic Systems. New York: Springer-Verlag, 1989.

[7] L. O. Chua, "Special issue on chaotic systems," Proc. IEEE. Aug. 1987.

[8] G. Dahlquist and A. Bjorck, Numerical Methods. Englewood-Cliffs, NJ: Prentice Hall, 1974.

[9] J. Guckenheimer and P. Holmes, Nonlinear Oscillations, Dynamical Systems, and Bifurcations of Vector Fields. New York: Springer-Verlag, 1983.

[10] D. Veitch, "The approach to constrained equations," Phys. Lett., vol. A 183, no. 4. pp. 319-326. Dec. 1993.

[11] O. E. Rossler, "Chaos in abstract kinetics: two prototypes," Bull. Mathemat. Biol., vol. 39. pp. 275-289. 1977.

[12] M. Diener, "On the Perfect Delay Convention" or "the Revolt of the Slaved Variables," in Chaos and Order in Nature. (Springer Series in Synergetics) 1981.

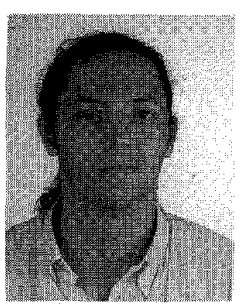

Enric Fossas received the Graduate and $\mathrm{Ph} . \mathrm{D}$. degrees in mathematics from Barcelona University, Spain, in 1981 and 1986, respectively.

Since 1981 he has taught mathematics at the Universitat de Barcelona and at the Universitat Politecnica de Catalunya, where he is employed as an assistant professor. His research interests are in the field of system theory, control and differential geometry.

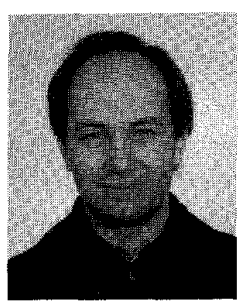

Gerard Olivar received the Graduate degree in mathematics from the Universitat de Barcelona in 1987.

Currently, he is working toward the $\mathrm{Ph} . \mathrm{D}$. degree in mathematics at the Universitat Politècnica de Catalunya, where he is a lecturer. His research interests include control theory and chaotic dynamics. 